Journal of Mathematics and Statistics 4 (1): 46-50, 2008

ISSN 1549-3644

(C) 2008 Science Publications

\title{
An Operator Defined by Convolution Involving the Polylogarithms Functions
}

K. Al Shaqsi and M. Darus

School of Mathematical Sciences, Faculty of Science and Technology

University Kebangsaan Malaysia, Bangi 43600 Selangor D. Ehsan, Malaysia

\begin{abstract}
We define an operator on the class $\mathcal{A}$ of analytic functions in the unit disk $\mathbb{U}=\{z:|z|<1\}$ involving the polylogarithms functions and introduce certain new subclasses of $\mathcal{A}$ using this operator. Some inclusion results, covering theorem, coefficients inequalities, and several other interesting properties of these classes are obtained.
\end{abstract}

Key words: Analytic functions, univalent functions, polylogarithms functions, derivative operato

\section{INTRODUCTION}

Let $\mathcal{A}$ denote the class of functions of the form:

$$
f(z)=z+\sum_{k=2}^{\infty} a_{k} z^{k}
$$

which are analytic in the unit disk $\mathbb{U}=\{z:|z|<1\}$. For functions $f$ given by (1) and $g(z)=z+\sum_{k=2}^{\infty} b_{k} z^{k}$, let $(f * g)(z)$ denote the Hadamard product (or convolution) of $f(z)$ and $\quad g(z), \quad$ defined by $(f * g)(z)=z+\sum_{k=2}^{\infty} a_{k} b_{k} z^{k}$. And for the functions $f(z)$ and $g(z)$ in $\mathcal{A}$, we say that $f$ is subordinate to $g$ in $\mathbb{U}$, and write $f \prec g$, if there exists a Schwarz function $w$ in $\mathcal{A}$ with $|w(z)|<1 \quad$ and $\quad w(0)=0 \quad$ such that $f(z)=g(w(z))$ in $\mathbb{U}$.

For $f \in \mathcal{A}$, Sălăgean ${ }^{[9]}$ has introduced the following operator called the Sălăgean operator:

$$
\begin{aligned}
& D^{0} f(z)=f(z), \\
& D^{1} f(z)=D f(z)=z f^{\prime}(z), \\
& D^{n} f(z)=D\left(D^{n-1} f(z)\right), \quad(n \in \mathbb{N}) .
\end{aligned}
$$

Note that

$$
\begin{aligned}
D^{n} f(z) & =z+\sum_{k=2}^{\infty} k^{n} a_{k} z^{k}, \\
\left(n \in \mathbb{N}_{0}\right. & =\mathbb{N} \bigcup\{0\}) .
\end{aligned}
$$

Let $f \in \mathcal{A}$. Denote by $D^{\lambda}: \mathcal{A} \rightarrow \mathcal{A}$, the operator defined by:

$$
D^{\lambda} f(z)=\frac{z}{(1-z)^{\lambda+1}} * f(z) \quad(\lambda>-1) .
$$

It is obvious that $D^{0} f(z)=f(z)$, $D^{1} f(z)=z f^{\prime}(z)$ and

$$
D^{\delta} f(z)=\frac{z\left(z^{\delta-1} f(z)\right)^{(\delta)}}{\delta !}, \quad\left(\delta \in \mathbb{N}_{0}\right) .
$$

Note that $D^{\delta} f(z)=z+\sum_{k=2}^{\infty} C(\delta, k) a_{k} z^{k}$, where $C(\delta, k)=\left(\begin{array}{c}k+\delta-1 \\ \delta\end{array}\right)$ and $\delta \in \mathbb{N}_{0}$.

The operator $D^{\delta} f$ is called the Ruscheweyh derivative operator $^{[8]}$.

Finally, let $P$ denote the class of functions of the form $p(z)=1+p_{1} z+p_{2} z^{2}+\cdots$ analytic in $\mathbb{U}$ which satisfy the condition $\operatorname{Re}\{p(z)\}>0$.

We recall here the definition of the wellknown generalization of the polylogarithm function $G(n ; z)$ given by

$$
G(n ; z)=\sum_{k=1}^{\infty} \frac{z^{k}}{k^{n}} \quad(n \in \square, z \in \mathbb{U}) .
$$

We note that $G(-1 ; z)=z /(1-z)^{2}$ is Koebe function. For more about polylogarithms in theory of univalent functions see Ponnusamy and Sabapathy ${ }^{[7]}$ and Ponnusamy ${ }^{[6]}$.

\section{Corresponding Author: M. Darus, Faculty of Science and Technology, School of Mathematical Sciences, Universiti} Kebangsaan Malaysia, Bangi 43600 Selangor D. Ehsan, Malaysia 
We now introduce a function $(G(n ; z))^{(-1)}$ given by

$G(n ; z) *(G(n ; z))^{(-1)}=\frac{z}{(1-z)^{\lambda+1}}$,

$(\lambda>-1, n \in \square)$

and obtain the following linear operator

$$
\mathfrak{D}_{\lambda}^{n} f(z)=(G(n ; z))^{(-1)} * f(z) .
$$

Now we find the explicit form of the function $(G(n ; z))^{(-1)}$. It is well known that for $\lambda>-1$ we have:

$\frac{z}{(1-z)^{\lambda+1}}=\sum_{k=0}^{\infty} \frac{(\lambda+1)_{k}}{k !} z^{k+1} \quad(z \in \mathbb{U})$.

Putting (3) and (5) in (4), we get:

$$
\sum_{k=1}^{\infty} \frac{1}{k^{n}} z^{k} *(G(n ; z))^{(-1)}=\sum_{k=1}^{\infty} \frac{(k+\lambda-1) !}{\lambda !(k-1) !} z^{k} .
$$

Therefore the function $(G(n ; z))^{(-1)}$ has the following form

$(G(n ; z))^{(-1)}=\sum_{k=1}^{\infty} k^{n} \frac{(k+\lambda-1) !}{\lambda !(k-1) !} z^{k} \quad(z \in \mathbb{U})$.

For $n, \lambda \in \mathbb{N}_{0}$, we note that

$\mathfrak{D}_{\lambda}^{\mathfrak{n}} f(z)=z+\sum_{k=2}^{\infty} k^{n} \frac{(k+\lambda-1) !}{\lambda !(k-1) !} z^{k} \quad(z \in \mathbb{U})$.

Note that $\mathfrak{D}_{0}^{\mathfrak{n}} \equiv D^{n}$ and $\mathfrak{D}_{\lambda}^{0} \equiv D^{\delta}$ which are Sălăgean and Ruscheweyh derivative operators , respectively ${ }^{[9,8]}$. It is clear that the operator $\mathfrak{D}_{\lambda}^{n}$ included two known derivative operators. Also note that $\mathfrak{D}_{0}^{0} f(z)=f(z) \quad$ and $\mathfrak{D}_{0}^{1} f(z)=\mathfrak{D}_{1}^{0} f(z)=z f^{\prime}(z)$.

Definition 1: Let $K_{\lambda}^{n}(\phi(z))$ be the class of functions $f \in \mathcal{A}$ for which

$$
\begin{gathered}
\frac{z\left(\mathfrak{D}_{\lambda}^{n} f(z)\right)^{\prime}}{\mathfrak{D}_{\lambda}^{n} f(z)} \prec \phi(z), \\
\left(n, \lambda \in \mathbb{N}_{0} ; \phi \in P ; z \in \mathbb{U}\right) .
\end{gathered}
$$

Definition 2: $\operatorname{Le} \phi(z)=(1+(1-2 \alpha) z) /(1-z)$, then $K_{\lambda}^{n}(\phi) \equiv R_{\lambda}^{n}(\alpha)$ be the class of functions $f \in \mathcal{A}$ for which

$$
\begin{gathered}
\operatorname{Re}\left\{\frac{z\left(\mathfrak{D}_{\lambda}^{n} f(z)\right)^{\prime}}{\mathfrak{D}_{\lambda}^{n} f(z)}\right\}>\alpha, \\
\left(n, \lambda \in \mathbb{N}_{0} ; 0 \leq \alpha<1 ; z \in \mathbb{U}\right) .
\end{gathered}
$$

Note that $K_{0}^{0}(\phi) \equiv S^{*}(\phi)$ were introduced and studied by $\mathrm{Ma}$ and Minda $^{[5]}, R_{\lambda}^{0}(\alpha) \equiv R_{\lambda}(\alpha)$ were studied by Ahuja $^{[1]}$ and $R_{0}^{n}(\alpha) \equiv R_{n}(\alpha)$ were studied by $\mathrm{Kadioğlu}^{[4]}$. Also for different choices of $n, \lambda$, and $\phi$, we obtain several subclasses of analytic functions investigated earlier by other authors.

Let $\mathcal{T}$ denote the subclass of $\mathcal{A}$ consisting of the functions that can be expressed in the form

$$
f(z)=z-\sum_{k=2}^{\infty}\left|a_{k}\right| z^{k} .
$$

Finally, we defined the class $\mathcal{M}_{\lambda}^{n}(\alpha)=R_{\lambda}^{n}(\alpha) \cap \mathcal{T}$. Note that $\mathcal{M}_{\lambda}^{n}(\alpha) \subset R_{\lambda}^{n}(\alpha)$.

In this paper, we investigate several inclusion properties for the classes $K_{\lambda}^{n}(\phi(z))$ associated with the operator $\mathfrak{D}_{\lambda}^{n}$. Some applications involving operator are also obtained. Also, we derive several interesting properties of functions belonging to the $\mathcal{M}_{\lambda}^{n}(\alpha)$ consisting of analytic and univalent functions with negative coefficients. Coefficient inequalities, distortion theorems and result on integral operators are also given.

\section{THE CLASSES $K_{\lambda}^{n}(\phi(z))$}

To derive our first theorem, we need the following lemma due to Eenigenburg et al. ${ }^{[3]}$.

Lemma 1: Let $\beta, v$ be complex numbers. Let $\phi \in P$ be convex univalent in $\mathbb{U}$ with $\phi(0)=1$ and $\quad \operatorname{Re}[\beta \phi(z)+v]>0, \quad z \in \mathbb{U}$. If $p(z)=1+p_{1} z+p_{2} z^{2}+\cdots$ is analytic in $\mathbb{U}$ with $p(0)=1$, then

$$
p(z)+\frac{z p^{\prime}(z)}{\beta \phi(z)+v} \prec \phi(z) \Rightarrow p(z) \prec \phi(z),(z \in \mathbb{U}) .
$$

Theorem 1: Let $n, \lambda \in \mathbb{N}_{0}$ and $\phi \in P$. Then

$$
K_{\lambda+1}^{n}(\phi) \subset K_{\lambda}^{n}(\phi) .
$$

Proof: Let $f \in K_{\lambda+1}^{n}(\phi)$ and set

$$
p(z)=\frac{z\left(\mathfrak{D}_{\lambda}^{n} f(z)\right)^{\prime}}{\mathfrak{D}_{\lambda}^{n} f(z)}
$$

where $p(z)$ analytic in $\mathbb{U}$ with $p(0)=1$.

One can easily verify the identity

$$
z\left(\mathfrak{D}_{\lambda}^{n} f(z)\right)^{\prime}=(\lambda+1) \mathfrak{D}_{\lambda+1}^{n} f(z)-\lambda \mathfrak{D}_{\lambda}^{n} f(z) .
$$


By using (11) in (10), we get

$$
(\lambda+1) \frac{\mathfrak{D}_{\lambda+1}^{n} f(z)}{\mathfrak{D}_{\lambda}^{n} f(z)}=p(z)+\lambda .
$$

Taking the logarithmic differentiation on both sides of (12) and multiplying by $z$, we have

$$
\frac{z\left(\mathfrak{D}_{\lambda+1}^{n} f(z)\right)^{\prime}}{\mathfrak{D}_{\lambda+1}^{n} f(z)}=p(z)+\frac{z p^{\prime}(z)}{p(z)+\lambda}(z \in \mathbb{U}) .
$$

Applying Lemma 1 to (13), it follows that $p \prec \phi$, that is $f \in K_{\lambda}^{n}(\phi)$. Therefore, we complete the proof of Theorem 1 .

Corollary 1: Let $n, \lambda \in \mathbb{N}_{0}$ and $\phi \in P$. Then $K_{\lambda+1}^{n+1}(\phi) \subset K_{\lambda}^{n}(\phi)$.

Theorem 2: Let the function $f \in K_{\lambda}^{n}(\phi)$ and let $c$ be real number such $c>-1$, then the function $F$ defined by

$$
F(z)=\frac{c+1}{z^{c}} \int_{0}^{z} t^{c-1} f(t) d t
$$

belongs to the class $K_{\lambda+1}^{n}(\phi)$.

Proof: Let $f \in K_{\lambda+1}^{n}(\phi)$. Then

$$
\frac{z\left(\mathfrak{D}_{\lambda+1}^{n} F(z)\right)^{\prime}}{\mathfrak{D}_{\lambda+1}^{n} F(z)} \prec \phi(z) .
$$

Set

$$
p(z)=\frac{z\left(\mathfrak{D}_{\lambda}^{n} F(z)\right)^{\prime}}{\mathfrak{D}_{\lambda}^{n} F(z)} .
$$

From the representation of $F(z)$, it follows that

$$
z\left(\mathfrak{D}_{\lambda}^{n} F(z)\right)^{\prime}=(c+1) \mathfrak{D}_{\lambda}^{n} f(z)-c \mathfrak{D}_{\lambda}^{n} F(z) .
$$

By using the same technique as in the proof of Theorem 1, we get

$$
\frac{z\left(\mathfrak{D}_{\lambda}^{n} f(z)\right)^{\prime}}{\mathfrak{D}_{\lambda}^{n} f(z)}=p(z)+\frac{z p^{\prime}(z)}{p(z)+c} .
$$

By applying Lemma 1 we obtain the required result.

\section{THE CLASSES $\mathcal{M}_{\lambda}^{n}(\alpha)$}

First, we provide a sufficient condition for a function $f$ analytic in $U$ to be in $\mathcal{M}_{\lambda}^{n}(\alpha)$.

\section{Coefficient estimates:}

Theorem 3: Let the function $f$ be defined by (9). Then $f \in \mathcal{M}_{\lambda}^{n}(\alpha)$ if and only if

$$
\sum_{k=2}^{\infty}(k-\alpha) k^{n} C(\lambda, k)\left|a_{k}\right| \leq 1-\alpha,
$$

where $n, \lambda \in \mathbb{N}_{0}$ and $C(\lambda, k)=\left(\begin{array}{c}k+\lambda-1 \\ \lambda\end{array}\right)$.

Proof: Assume that the inequality (17) holds true and $|z|=1$. Then we obtain

$$
\begin{aligned}
\left|\frac{z\left(\mathfrak{D}_{\lambda}^{n} f(z)\right)^{\prime}}{\mathfrak{D}_{\lambda}^{n} f(z)}-1\right| & =\left|\frac{\sum_{k=2}^{\infty}(k-1) k^{n} C(\lambda, k) a_{k} z^{k}}{z-\sum_{k=2}^{\infty} k^{n} C(\lambda, k) a_{k} z^{k}}\right| \\
& \leq \frac{\sum_{k=2}^{\infty}(k-1) k^{n} C(\lambda, k)\left|a_{k}\right|}{1-\sum_{k=2}^{\infty} k^{n} C(\lambda, k)\left|a_{k}\right|} \\
& \leq 1-\alpha .
\end{aligned}
$$

This show that the values of $\frac{z\left(\mathfrak{D}_{\lambda}^{n} f(z)\right)^{\prime}}{\mathfrak{D}_{\lambda}^{n} f(z)}$ lies in a circle centered at $w=1$ whose radius 1 whose radius $1-\alpha$. Hence $f$ satisfies the condition (17).

Conversely, we assume that the function $f$ defined by (9) is in the class $\mathcal{M}_{\lambda}^{n}(\alpha)$. Then

$$
\operatorname{Re}\left\{\frac{z\left(\mathfrak{D}_{\lambda}^{n} f(z)\right)^{\prime}}{\mathfrak{D}_{\lambda}^{n} f(z)}\right\}=\operatorname{Re}\left\{\frac{z-\sum_{k=2}^{\infty} k^{n+1} C(\lambda, k) a_{k} z^{k}}{z-\sum_{k=2}^{\infty} k^{n} C(\lambda, k) a_{k} z^{k}}\right\}>\alpha .
$$

For $z \in \mathbb{U}$, we choose values of $z$ on the real axis so that $\frac{z\left(\mathfrak{D}_{\lambda}^{n} f(z)\right)^{\prime}}{\mathfrak{D}_{\lambda}^{n} f(z)}$ is real.

Upon clearing the denominator in (18) and letting $z \rightarrow 1^{-}$through real values, we obtain

$1-\sum_{k=2}^{\infty} k^{n+1} C(\lambda, k)\left|a_{k}\right| \geq \alpha\left\{1-\sum_{k=2}^{\infty} k^{n} C(\lambda, k)\left|a_{k}\right|\right\}$ which gives (17).

Finally the result is sharp with the extremal function $f$ given by

$$
\begin{gathered}
f(z)=z-\frac{1-\alpha}{(k-\alpha) k^{n} C(\lambda, k)} z^{k}, \\
\left(n, \lambda \in \mathbb{N}_{0} ; 0 \leq \alpha<1 ; k \geq 2\right) .
\end{gathered}
$$

Corollary 2: Let the function $f$ defined by (9) be in the class $\mathcal{M}_{\lambda}^{n}(\alpha)$. Then we have

$$
a_{k} \leq \frac{1-\alpha}{(k-\alpha) k^{n} C(\lambda, k)} \quad\left(n, \lambda \in \mathbb{N}_{0} ; 0 \leq \alpha<1 ; k \geq 2\right) \text {. }
$$

This equality is attained for the function $f$ given by (20). 


\section{Distortion theorem:}

A distortion property for function $f$ to be in the class $\mathcal{M}_{\lambda}^{n}(\alpha)$ given as follows:

Theorem 4: Let the function $f$ defined by (9) be in the class $\mathcal{M}_{\lambda}^{n}(\alpha)$. Then for $|z|=r$ we have

$r-\frac{1-\alpha}{(2-\alpha) 2^{n}(\lambda+1)} r^{2} \leq|f(z)| \leq r+\frac{1-\alpha}{(2-\alpha) 2^{n}(\lambda+1)} r^{2}$,

and

$1-\frac{1-\alpha}{(2-\alpha) 2^{n-1}(\lambda+1)} r \leq\left|f^{\prime}(z)\right| \leq r+\frac{1-\alpha}{(2-\alpha) 2^{n-1}(\lambda+1)} r$.

Proof: In view of Theorem 4, we have

$\sum_{k=2}^{\infty} a_{k} \leq \frac{1-\alpha}{(2-\alpha) 2^{n}(\lambda+1)}$.

Hence

$|f(z)| \leq r+\sum_{k=2}^{\infty}\left|a_{k}\right| r^{k} \leq r+\frac{1-\alpha}{(2-\alpha) 2^{n}(\lambda+1)} r^{2}$,

and

$|f(z)| \geq r-\sum_{k=2}^{\infty}\left|a_{k}\right| r^{k} \geq r-\frac{1-\alpha}{(2-\alpha) 2^{n}(\lambda+1)} r^{2}$.

In the same way we have

$1-\frac{1-\alpha}{(2-\alpha) 2^{n-1}(\lambda+1)} r \leq\left|f^{\prime}(z)\right| \leq r+\frac{1-\alpha}{(2-\alpha) 2^{n-1}(\lambda+1)} r$.

This completes the proof of the theorem. The above bounds are sharp. Equalities are attended for the following function

$f(z)=z-\frac{1-\alpha}{(2-\alpha) 2^{n}(\lambda+1)} z^{2}, \quad z= \pm r$.

Corollary 3: The disk $|z|<1$ is mapped onto a domain that contains the disk

$$
|w|<1-\frac{1-\alpha}{(2-\alpha) 2^{n}(\lambda+1)} .
$$

The result is sharp with extremal function (23).

Proof: The result follows upon letting $r \rightarrow 1$ in (22).

\section{Integral Operator:}

Bernardi $^{[5]}$ introduced integral operator defined as follows:

Let $f \in \mathcal{A}$ and $c>-1$. Then, for $z \in \mathbb{U}$

$$
F(z)=\frac{c+1}{z^{c}} \int_{0}^{z} t^{c-1} f(t) d t
$$

Now we consider our results.

Theorem 5: Let the function $f$ defined by (9) be in the class $\mathcal{M}_{\lambda}^{n}(\alpha)$ and let $c$ be real number such that $c>-1$, then the function $F$ defined by

$$
F(z)=\frac{c+1}{z^{c}} \int_{0}^{z} t^{c-1} f(t) d t
$$

Proof: From the representation of $F(z)$, it follows that

$$
F(z)=z-\sum_{k=2}^{\infty}\left|b_{k}\right| z^{k}
$$

where $\left|b_{k}\right|=\left(\frac{c+1}{c+k}\right)\left|a_{k}\right|<1$. Therefore

$\sum_{k=2}^{\infty}(k-\alpha) k^{n} C(\lambda, k)\left|b_{k}\right|$

$=\sum_{k=2}^{\infty}(k-\alpha) k^{n} C(\lambda, k)\left(\frac{c+1}{c+k}\right)\left|a_{k}\right|$

$\leq \sum_{k=2}^{\infty}(k-\alpha) k^{n} C(\lambda, k)\left|a_{k}\right| \leq 1-\alpha$.

Since $f \in \mathcal{M}_{\lambda}^{n}(\alpha)$ and hence by Theorem 5, $F \in \mathcal{M}_{\lambda}^{n}(\alpha)$.

\section{ACKNOWLEDGMENT}

The work here was supported by SAGA: STGL012-2006, Academy of Sciences, Malaysia.

\section{REFERENCES}

1. Ahuja, O., 1985. Integral operators of certain univalent functions, Internat. J. Math. Sci., 8: 653-662.

2. Bernardi, S. D., 1969. Convex and starlike univalent functions. Trans. Amer. Math. Soc., 135: 429-446.

3. Eenigenburg, P., Miller, S. S., Mocanu, P. T. and M. O. Reade, 1983. On a BriotBouquet differential subordination. General Inequal., 3: 339-348.

4. Kadioğlu, E., 2003. On subclass of univalent functions with negative coefficients. Appl. Math. Comput., 146: 351-358.

5. Ma, W. and D. Minda, 1994. A unified treatment of some special classes of univalent functions, in: Proceedings of the conference on complex analysis, Li, Z., Ren, F., Yang, L., and Zhang, S. (Eds.), Int. Press , 157-169. 
6. Ponnusamy, S., 1998. Inclusion theorems for convolution product of second order polylogarithms and functions with the derivative in a half plane. Rocky Mountain J. Math., 28(2): 695-733.

7. Ponnusamy, S. and S. Sabapathy, 1996. Polylogarithms in the theory of univalent functions. Results in Mathematics, 30:136150.
8. Ruscheweyh, St., 1975. New criteria for univalent functions. Proc. Amer. Math. Soc., 49: 109-115.

9. Sălăgean, G. S., 1983. Subclasses of univalent functions. Lecture Notes in Math.(Springer-Verlag), 1013: 362-372. 